\title{
Multidegrees of Tame Automorphisms in Dimension Three
}

\author{
by \\ Xiaosong Sun and Yan CHen
}

\begin{abstract}
We discuss when a sequence of positive integers can be the multidegree of some tame automorphism in dimension three, and we also relate these investigations to the problem of whether there exists a tame automorphism admitting a reduction of type II or type III.
\end{abstract}

2010 Mathematics Subject Classification: Primary 14R10.

Keywords: polynomial maps, tame automorphisms, multidegrees.

\section{$\S 1$. Introduction}

Throughout this paper, $k$ is a field of characteristic zero and $\mathbb{N}$ is the set of non-negative integers. A map $F=\left(F_{1}, \ldots, F_{n}\right): k^{n} \rightarrow k^{n}$ of the form $\alpha \mapsto$ $\left(F_{1}(\alpha), \ldots, F_{n}(\alpha)\right)$ is called a polynomial map if $F_{i} \in k\left[X_{1}, \ldots, X_{n}\right], 1 \leq i \leq n$. A polynomial map is called an automorphism if it has an inverse which is also a polynomial map.

An automorphism of the form $\left(X_{1}, \ldots, X_{i-1}, c X_{i}+a, X_{i+1}, \ldots, X_{n}\right)$ is called elementary if $0 \neq c \in k$ and $a$ is a polynomial not containing $X_{i}$. A finite composition of elementary automorphisms is called tame. The famous Tame Generators Problem asks if every polynomial automorphism is tame. It has an affirmative answer in dimension 2 (known as the Jung-van der Kulk theorem, see [J, Kul] or [E, Section 5.1]) and has a negative answer in dimension 3 (Shestakov and Umirbaev [SU1, SU2]). It remains open for any dimension $n \geq 4$.

Define by $\operatorname{tdeg} F:=\sum_{i=1}^{n} \operatorname{deg} F_{i}$ the total degree of a polynomial map $F$. An automorphism $F$ is said to admit an elementary reduction if there exists an

Communicated by S. Mukai. Received February 16, 2011.

X. Sun: School of Mathematical Sciences, Peking University, Beijing 100871, China, and School of Mathematics, Jilin University, Changchun 130012, China; e-mail: sunxs@pku.edu.cn

Y. Chen: School of Mathematics, Jilin University, Changchun 130012, China; e-mail: cy_185@163.com 
elementary automorphism $E \operatorname{such}$ that $\operatorname{tdeg}(E \circ F)<\operatorname{tdeg} F$, where o denotes composition. In dimension 3, four types of non-elementary reductions, labeled I-IV, were defined by Shestakov and Umirbaev ([SU2, Definition 1-4]), who showed that every tame automorphism $F: k^{3} \rightarrow k^{3}$ with $\operatorname{tdeg} F>3$ admits an elementary reduction or a reduction of one of the types I-IV ([SU2, Theorem 2]). They observed that an automorphism given by Nagata $[\mathrm{N}]$ admits none of these reductions, and thus is not tame.

There exists a tame automorphism admitting a reduction of type I (see [SU2, Example 1], and [EMW] for more examples). But recently, Kuroda [Kur2, Theorem 7.1] showed that there does NOT exist a tame automorphism admitting a reduction of type IV. However it is still open whether there exists a tame automorphism admitting a reduction of type II or III.

Karaś [K2] proposed the following problem: define by mdeg $F:=\left(\operatorname{deg} F_{1}, \ldots\right.$, $\left.\operatorname{deg} F_{n}\right)$ the multidegree of a polynomial map $F$ and by $\operatorname{mdeg}\left(T\left(k^{n}\right)\right)$ the set of multidegrees of tame automorphisms from $k^{n}$ to $k^{n}$. Which sequences $\left(d_{1}, \ldots, d_{n}\right)$ belong to $\operatorname{mdeg}\left(T\left(k^{n}\right)\right)$ ?

It is well known that $\left(d_{1}, d_{2}\right) \in \operatorname{mdeg}\left(T\left(k^{2}\right)\right)$ if and only if $d_{1} \mid d_{2}$ or $d_{2} \mid d_{1}$ (see for example [E, Section 5.1]). In dimension 3, some partial results were obtained by Karaś in [K1, K2] as follows.

Theorem 1.1 (Karaś). (i) ([K2, Theorem 1.1]) Let $3 \leq d_{2} \leq d_{3}$ be integers. Then $\left(3, d_{2}, d_{3}\right) \in \operatorname{mdeg}\left(T\left(k^{3}\right)\right)$ if and only if $3 \mid d_{2}$ or $d_{3} \in 3 \mathbb{N}+d_{2} \mathbb{N}$.

(ii) ([K1, Theorem 1]) Let $3 \leq p_{1} \leq d_{2} \leq d_{3}$ be integers. If $p_{1}$ and $d_{2}$ are prime numbers, then $\left(p_{1}, d_{2}, d_{3}\right) \in \operatorname{mdeg}\left(T\left(k^{3}\right)\right)$ if and only if $d_{3} \in p_{1} \mathbb{N}+d_{2} \mathbb{N}$.

These investigations led to the following conjecture.

Conjecture 1.2 ([K2, Conjecture 4.1]). Let $3 \leq p_{1} \leq d_{2} \leq d_{3}$ be integers with $p_{1}$ a prime number. Then $\left(p_{1}, d_{2}, d_{3}\right) \in \operatorname{mdeg}\left(T\left(k^{3}\right)\right)$ if and only if $p_{1} \mid d_{2}$ or $d_{3} \in$ $p_{1} \mathbb{N}+d_{2} \mathbb{N}$

In this paper, we show that Conjecture 1.2 holds if additionally one of the following conditions is satisfied (i) $d_{2} / \operatorname{gcd}\left(d_{2}, d_{3}\right) \neq 2$; (ii) $d_{3} / \operatorname{gcd}\left(d_{2}, d_{3}\right) \neq 3$; (iii) $d_{2} \geq 2 p_{1}-5$. As corollaries, we show that Conjecture 1.2 holds in the following cases: (1) $d_{2}$ is odd; (2) $p_{1}=3$ or 5 ; (3) $p_{1}=7$ and $\left(d_{2}, d_{3}\right) \neq(8,12)$. Furthermore, we relate the investigations with the problem of whether there exists a tame automorphism admitting a reduction of type II or III. We show that, if $(7,8,12) \in \operatorname{mdeg}\left(T\left(k^{3}\right)\right)$, then there exists a tame automorphism admitting a reduction of type II, and if $\left(p_{1}, 2 p_{1}-6,3 p_{1}-9\right) \in \operatorname{mdeg}\left(T\left(k^{3}\right)\right)$, where $p_{1}>7$ is a prime number, then there exists one admitting a reduction of type III. 


\section{$\S 2$. Preliminaries}

In this section, we recall some notions and results about the Poisson bracket and *-reduced pair; for details, see [SU1, SU2].

Let $L\left\langle X_{1}, \ldots, X_{n}\right\rangle$ be the free Lie algebra with free generators $X_{1}, \ldots, X_{n}$. Let $P L\left\langle X_{1}, \ldots, X_{n}\right\rangle$ be the free Poisson algebra with free generators $X_{1}, \ldots, X_{n}$, which is the $k$-algebra generated by a linear basis of $L\left\langle X_{1}, \ldots, X_{n}\right\rangle$ and the Poisson bracket of which is induced by the Lie bracket of $L\left\langle X_{1}, \ldots, X_{n}\right\rangle$. It becomes a graded algebra if we put $\operatorname{deg} X_{i}=1, \operatorname{deg}\left[X_{i}, X_{j}\right]=2, i \neq j$, etc. Imbedding the polynomial algebra $k\left[X_{1}, \ldots, X_{n}\right]$ into $P L\left\langle X_{1}, \ldots, X_{n}\right\rangle$, one can define the Poisson bracket of two polynomials $f, g$ to be

$$
[f, g]=\sum_{1 \leq i<j \leq n}\left(\frac{\partial f}{\partial X_{i}} \frac{\partial g}{\partial X_{j}}-\frac{\partial f}{\partial X_{j}} \frac{\partial g}{\partial X_{i}}\right)\left[X_{i}, X_{j}\right] .
$$

Hence

$$
\operatorname{deg}[f, g]=2+\max _{1 \leq i<j \leq n} \operatorname{deg}\left(\frac{\partial f}{\partial X_{i}} \frac{\partial g}{\partial X_{j}}-\frac{\partial f}{\partial X_{j}} \frac{\partial g}{\partial X_{i}}\right) .
$$

Note that $\operatorname{deg}[f, g] \geq 2$ if $f, g$ are algebraically independent.

Definition 2.1 ([SU1, Definition 1]). A pair of polynomials $f, g \in k\left[X_{1}, \ldots, X_{n}\right]$ is called ${ }^{*}$-reduced if

(1) $f, g$ are algebraically independent;

(2) $\bar{f}, \bar{g}$ are algebraically dependent, where $\bar{h}$ denotes the highest homogeneous part of $h$;

(3) $\bar{f} \notin k[\bar{g}]$ and $\bar{g} \notin k[\bar{f}]$.

Let $f, g$ be a ${ }^{*}$-reduced pair with $\operatorname{deg} f \leq \operatorname{deg} g$ and let $p=\frac{\operatorname{deg} f}{\operatorname{gcd}(\operatorname{deg} f, \operatorname{deg} g)}$. Then $f, g$ is also called a $p$-reduced pair.

Theorem 2.2 ([SU1, Theorem 3]). Let $f, g$ be a p-reduced pair and let $G(x, y) \in$ $k[x, y]$ with $\operatorname{deg}_{y} G(x, y)=p q+r, 0 \leq r<p$. Then

$$
\operatorname{deg} G(f, g) \geq q(p \operatorname{deg} g-\operatorname{deg} g-\operatorname{deg} f+\operatorname{deg}[f, g])+r \operatorname{deg} g .
$$

Remark 2.3. Karaś observed that Theorem 2.2 is also true if the second condition of Definition 2.1 is not satisfied (see [K2, Proposition 2.4]).

We close this section by recalling several results which will also be used in the next section.

Lemma 2.4 (Brauer $[\mathrm{B}])$. If $a, b$ are positive integers such that $\operatorname{gcd}(a, b)=1$, then $l \in a \mathbb{N}+b \mathbb{N}$ for every integer $l \geq(a-1)(b-1)$. 
Lemma 2.5 ([K2, Proposition 2.2]). Let $d_{1} \leq \cdots \leq d_{n}$ be positive integers. If there exists some $i$ such that $d_{i} \in d_{1} \mathbb{N}+\cdots+d_{i-1} \mathbb{N}$, then $\left(d_{1}, \ldots, d_{n}\right) \in$ $\operatorname{mdeg}\left(T\left(k^{n}\right)\right)$.

\section{§3. Multidegrees of tame automorphisms of $k^{3}$}

We start with a lemma.

Lemma 3.1. Let $F=\left(F_{1}, F_{2}, F_{3}\right)$ be an automorphism with $\operatorname{mdeg} F=\left(d_{1}, d_{2}, d_{3}\right)$. If $\operatorname{deg}\left[F_{s}, F_{t}\right]=2$, then $d_{s} \mid d_{t}$ or $d_{t} \mid d_{s}$, where $1 \leq s<t \leq 3$.

Proof. Let $T$ be a linear automorphism and $F^{\prime}=F \circ T$. It is easy to verify that $\operatorname{deg}\left[F_{s}^{\prime}, F_{t}^{\prime}\right]=\operatorname{deg}\left[F_{s}, F_{t}\right]$ for any $1 \leq s<t \leq 3$. Replacing $F$ by some $F \circ T$ if necessary, we may assume that $F=\left(X_{1}+H_{1}, X_{2}+H_{2}, X_{3}+H_{3}\right)$, where each $H_{i}$ contains no linear terms.

Suppose that $\operatorname{deg}\left[F_{s}, F_{t}\right]=2$ for some $1 \leq s<t \leq 3$, say $\operatorname{deg}\left[F_{2}, F_{3}\right]=2$. Since

$$
\operatorname{deg}\left[F_{2}, F_{3}\right]=2+\max _{1 \leq i<j \leq 3} \operatorname{deg} c_{i j}, \quad \text { where } \quad c_{i j}=\frac{\partial F_{2}}{\partial X_{i}} \frac{\partial F_{3}}{\partial X_{j}}-\frac{\partial F_{3}}{\partial X_{i}} \frac{\partial F_{2}}{\partial X_{j}},
$$

we have $c_{i j} \in k, 1 \leq i<j \leq 3$. It follows that

$$
c_{12}=\frac{\partial H_{2}}{\partial X_{1}} \frac{\partial H_{3}}{\partial X_{2}}-\frac{\partial H_{3}}{\partial X_{1}}\left(1+\frac{\partial H_{2}}{\partial X_{2}}\right)=0, \quad c_{13}=\frac{\partial H_{2}}{\partial X_{1}}\left(1+\frac{\partial H_{3}}{\partial X_{3}}\right)-\frac{\partial H_{3}}{\partial X_{1}} \frac{\partial H_{2}}{\partial X_{3}}=0 .
$$

Notice that $\partial H_{2} / \partial X_{1}=0$ if and only if $\partial H_{3} / \partial X_{1}=0$. Now suppose that $\partial H_{2} / \partial X_{1} \neq 0$. Then $\partial H_{3} / \partial X_{1} \neq 0$. Let $u$ and $v$ be the lowest homogeneous parts of $\partial H_{2} / \partial X_{1}$ and $\partial H_{3} / \partial X_{1}$ respectively. If $\operatorname{deg} u \leq \operatorname{deg} v$, then $u$ is the lowest homogeneous part of $c_{13}$, which contradicts $c_{13}=0$. Similarly, if $\operatorname{deg} v \leq \operatorname{deg} u$, then $v$ is the lowest homogeneous part of $c_{12}$, which contradicts $c_{12}=0$.

Therefore, $\partial H_{2} / \partial X_{1}=0$ and $\partial H_{3} / \partial X_{1}=0$. It follows that $\left(F_{2}, F_{3}\right)$ is an automorphism in dimension 2 , and thus $d_{2} \mid d_{3}$ or $d_{3} \mid d_{2}$.

Lemma 3.2. Let $3 \leq p_{1} \leq d_{2} \leq d_{3}$ be integers such that $p_{1}$ is a prime number, $p_{1} \nmid d_{2}$ and $d_{3} \notin p_{1} \mathbb{N}+d_{2} \mathbb{N}$. If $\left(p_{1}, d_{2}, d_{3}\right) \in \operatorname{mdeg}\left(T\left(k^{3}\right)\right)$, then there exists a tame automorphism with multidegree $\left(p_{1}, d_{2}, d_{3}\right)$ which admits an elementary reduction.

Proof. If $\left(p_{1}, d_{2}, d_{3}\right) \in \operatorname{mdeg}\left(T\left(k^{3}\right)\right)$, then there exists a tame automorphism $F$ with mdeg $F=\left(p_{1}, d_{2}, d_{3}\right)$. By [SU2, Theorem 2] and [Kur2, Theorem 7.1], $F$ admits an elementary reduction or a reduction of one of the types I-III.

By [SU2, Definitions 1 and 2], if there exists a tame automorphism admitting a reduction of type I or II, then there exists a tame automorphism admitting an elementary reduction with the same multidegree. 
Now suppose that $F$ admits a reduction of type III. Then by [SU2, Definition 3] (through a permutation of indices), there exists some positive integer $m$ such that one of the following is satisfied:

$$
\begin{aligned}
& m<\operatorname{deg} F_{1}=p_{1} \leq \frac{3}{2} m, \quad \operatorname{deg} F_{2}=d_{2}=2 m, \quad \operatorname{deg} F_{3}=d_{3}=3 m \\
& \operatorname{deg} F_{1}=p_{1}=\frac{3}{2} m, \quad \operatorname{deg} F_{2}=d_{2}=2 m, \quad \frac{5}{2} m<\operatorname{deg} F_{3}=d_{3} \leq 3 m
\end{aligned}
$$

In the case (3.2), the condition that $p_{1}$ is a prime number implies that $m=2$, $p_{1}=3$ and $d_{3}=6$, which contradicts $d_{3} \notin p_{1} \mathbb{N}+d_{2} \mathbb{N}$. So it suffices to consider the case (3.1).

Also by [SU2, Definition 3], there exist $\alpha, \beta, \gamma \in k$ with $(\alpha, \beta, \gamma) \neq(0,0,0)$ such that the elements $G_{2}:=F_{2}-\beta F_{1}, G_{3}:=F_{3}-\gamma F_{1}-\alpha F_{1}^{2}$ satisfy $\operatorname{deg} G_{2}=2 m$, $\operatorname{deg} G_{3}=3 m$ and $E \circ\left(F_{1}, G_{2}, G_{3}\right)=\left(G_{1}, G_{2}, G_{3}\right)$, for some elementary automorphism $E$, with $\operatorname{deg} G_{1} \leq \frac{3}{2} m, \operatorname{deg}\left[G_{1}, G_{2}\right]<3 m+\operatorname{deg}\left[G_{2}, G_{3}\right]$ and $\operatorname{deg} G_{1}<$ $m+\operatorname{deg}\left[G_{2}, G_{3}\right]$. By $\left[\mathrm{SU} 2\right.$, Corollary 4], $\operatorname{tdeg}\left(G_{1}, G_{2}, G_{3}\right)<\operatorname{tdeg} F$. Since tdeg $F=$ $\operatorname{tdeg}\left(F_{1}, G_{2}, G_{3}\right)$, it follows that $\left(F_{1}, G_{2}, G_{3}\right)$ admits an elementary reduction and $\operatorname{mdeg}\left(F_{1}, G_{2}, G_{3}\right)=\left(p_{1}, d_{2}, d_{3}\right)$.

Theorem 3.3. Let $3 \leq p_{1} \leq d_{2} \leq d_{3}$ be integers with $p_{1}$ a prime number. If one of the following conditions is satisfied: (i) $d_{2} / \operatorname{gcd}\left(d_{2}, d_{3}\right) \neq 2$; (ii) $d_{3} / \operatorname{gcd}\left(d_{2}, d_{3}\right) \neq 3$; (iii) $d_{2} \geq 2 p_{1}-5$, then $\left(p_{1}, d_{2}, d_{3}\right) \in \operatorname{mdeg}\left(T\left(k^{3}\right)\right)$ if and only if $p_{1} \mid d_{2}$ or $d_{3} \in$ $p_{1} \mathbb{N}+d_{2} \mathbb{N}$.

Proof. By Lemma 2.5, if $p_{1} \mid d_{2}$ or $d_{3} \in p_{1} \mathbb{N}+d_{2} \mathbb{N}$, then $\left(p_{1}, d_{2}, d_{3}\right) \in \operatorname{mdeg}\left(T\left(k^{3}\right)\right)$. Now assume that $p_{1} \nmid d_{2}$ and $d_{3} \notin p_{1} \mathbb{N}+d_{2} \mathbb{N}$. By Lemma 3.2, we only need to show that, if one of the three conditions in the theorem is satisfied, then an automorphism $F$ with mdeg $F=\left(p_{1}, d_{2}, d_{3}\right)$ does not admit an elementary reduction.

Since $p_{1} \nmid d_{2}$ and $d_{3} \notin p_{1} \mathbb{N}+d_{2} \mathbb{N}$, we have $d_{3}<\left(p_{1}-1\right)\left(d_{2}-1\right)$ due to Lemma 2.4. Moreover Lemma 3.1 yields $\operatorname{deg}\left[F_{s}, F_{t}\right] \geq 3$ for any $1 \leq s<t \leq 3$.

(1) Suppose that $\left(F_{1}, F_{2}, F_{3}-g\left(F_{1}, F_{2}\right)\right)$ is an elementary reduction of $F$, i.e., $\operatorname{deg}\left(F_{3}-g\left(F_{1}, F_{2}\right)\right)<\operatorname{deg} F_{3}$, where $g \in k[x, y]$. Then $\operatorname{deg} g\left(F_{1}, F_{2}\right)=\operatorname{deg} F_{3}=d_{3}$. Notice that $p_{1} / \operatorname{gcd}\left(p_{1}, d_{2}\right)=p_{1}$. Let $\operatorname{deg}_{y} g(x, y)=q p_{1}+r$, where $0 \leq r<p_{1}$. The pair $F_{1}, F_{2}$ satisfies the first and the third condition of Definition 2.1, since $F_{1}, F_{2}$ are algebraically independent and $p_{1} \nmid d_{2}$. Then by Theorem 2.2 (and noticing Remark 2.3, similarly hereinafter), we have

$$
\begin{aligned}
d_{3} & =\operatorname{deg} g\left(F_{1}, F_{2}\right) \geq q\left(p_{1} d_{2}-d_{2}-p_{1}+\operatorname{deg}\left[F_{1}, F_{2}\right]\right)+r d_{2} \\
& \geq q\left(p_{1} d_{2}-d_{2}-p_{1}+3\right)+r d_{2} \geq q\left(p_{1}-1\right)\left(d_{2}-1\right)+r d_{2} .
\end{aligned}
$$

Since $d_{3}<\left(p_{1}-1\right)\left(d_{2}-1\right)$, we have $q=0$ and thus $\operatorname{deg}_{y} g(x, y)=r<p_{1}$. 
Let $g(x, y)=\sum_{i=0}^{p_{1}-1} g_{i}(x) y^{i}$. Since $\operatorname{gcd}\left(p_{1}, d_{2}\right)=1$, the sets $p_{1} \mathbb{N}, d_{2}+p_{1} \mathbb{N}, \ldots$, $\left(p_{1}-1\right) d_{2}+p_{1} \mathbb{N}$ are disjoint. Hence

$$
\begin{aligned}
d_{3} & =\operatorname{deg} g\left(F_{1}, F_{2}\right)=\operatorname{deg}\left(\sum_{i=0}^{p_{1}-1} g_{i}\left(F_{1}\right) F_{2}^{i}\right) \\
& =\max _{0 \leq i \leq p_{1}-1}\left(\operatorname{deg} F_{1} \operatorname{deg} g_{i}+i \operatorname{deg} F_{2}\right)=\max _{0 \leq i \leq p_{1}-1}\left(p_{1} \operatorname{deg} g_{i}+i d_{2}\right),
\end{aligned}
$$

which contradicts $d_{3} \notin p_{1} \mathbb{N}+d_{2} \mathbb{N}$.

(2) Suppose that $\left(F_{1}, F_{2}-g\left(F_{1}, F_{3}\right), F_{3}\right)$ is an elementary reduction of $F$, where $g \in k[x, y]$. Then $\operatorname{deg} g\left(F_{1}, F_{3}\right)=\operatorname{deg} F_{2}=d_{2}$. Notice that $p_{1} / \operatorname{gcd}\left(p_{1}, d_{3}\right)$ $=p_{1}$. Let $\operatorname{deg}_{y} g(x, y)=q p_{1}+r$, where $0 \leq r<p_{1}$. By Theorem 2.2,

$$
\begin{aligned}
d_{2}=\operatorname{deg} g\left(F_{1}, F_{3}\right) & \geq q\left(p_{1} d_{3}-d_{3}-p_{1}+\operatorname{deg}\left[F_{1}, F_{3}\right]\right)+r d_{3} \\
& \geq q\left(p_{1} d_{3}-2 d_{3}\right)+r d_{3} \geq q d_{3}+r d_{3},
\end{aligned}
$$

which implies that $q=r=0$. Then $\operatorname{deg}_{y} g(x, y)=0$ and thus $d_{2}=\operatorname{deg} g\left(F_{1}, F_{3}\right)=$ $\operatorname{deg} g\left(F_{1}\right) \in p_{1} \mathbb{N}$, which contradicts $p_{1} \nmid d_{2}$.

(3) Suppose that $\left(F_{1}-g\left(F_{2}, F_{3}\right), F_{2}, F_{3}\right)$ is an elementary reduction of $F$, where $g \in k[x, y]$. Then $\operatorname{deg} g\left(F_{2}, F_{3}\right)=\operatorname{deg} F_{1}=p_{1}$. Let $p=d_{2} / \operatorname{gcd}\left(d_{2}, d_{3}\right)$ and let $\operatorname{deg}_{y} g(x, y)=q p+r$, where $0 \leq r<p$. By Theorem 2.2 we obtain

$$
p_{1}=\operatorname{deg} g\left(F_{2}, F_{3}\right) \geq q\left(p d_{3}-d_{3}-d_{2}+\operatorname{deg}\left[F_{2}, F_{3}\right]\right)+r d_{3} .
$$

If $\operatorname{deg}_{y} g(x, y)=0$, then $p_{1}=\operatorname{deg} g\left(F_{2}, F_{3}\right)=\operatorname{deg} g\left(F_{2}\right) \in d_{2} \mathbb{N}$, a contradiction. So in what follows we always assume that $\operatorname{deg}_{y} g(x, y)>0$.

We divide the following discussion into several subcases.

(a) $p=d_{2} / \operatorname{gcd}\left(d_{2}, d_{3}\right) \neq 2$ or $d_{3} / \operatorname{gcd}\left(d_{2}, d_{3}\right) \neq 3$.

If $p \neq 2$, noticing that $p>1$, we have $p \geq 3$. Then

$$
p d_{3}-d_{3}-d_{2}+\operatorname{deg}\left[F_{2}, F_{3}\right] \geq 2 d_{3}-d_{2} \geq d_{2} \text {. }
$$

If $p=2$, then $d_{3} / \operatorname{gcd}\left(d_{2}, d_{3}\right) \neq 3$. Let $\operatorname{gcd}\left(d_{2}, d_{3}\right)=m$. Then $d_{2}=2 m, d_{3}=$ $l m$, where $l \geq 4$, and thus $d_{3}-d_{2} \geq d_{2}$. We have also

$$
p d_{3}-d_{3}-d_{2}+\operatorname{deg}\left[F_{2}, F_{3}\right] \geq d_{3}-d_{2} \geq d_{2} .
$$

Then it follows by $(3.3)$ that $p_{1} \geq q d_{2}+r d_{3}$, which implies that $q=r=0$. Hence $\operatorname{deg}_{y} g(x, y)=0$, a contradiction.

(b) $d_{2} \geq 2 p_{1}-5$.

By (a) we may assume that $p=d_{2} / \operatorname{gcd}\left(d_{2}, d_{3}\right)=2$ and $d_{3} / \operatorname{gcd}\left(d_{2}, d_{3}\right)=3$, which implies that $d_{3}-d_{2} \geq d_{2} / 2$. Due to Lemma 3.1 , we have $\operatorname{deg}\left[F_{2}, F_{3}\right] \geq 3$. 
Then by (3.3) we obtain

$$
\begin{aligned}
p_{1} & \geq q\left(2 d_{3}-d_{3}-d_{2}+\operatorname{deg}\left[F_{2}, F_{3}\right]\right)+r d_{3} \\
& \geq q\left(d_{2} / 2+3\right)+r d_{3} \geq q\left(p_{1}+1 / 2\right)+r d_{3} .
\end{aligned}
$$

It follows that $q=r=0$, and thus $\operatorname{deg}_{y} g(x, y)=0$, also a contradiction.

It is easy to verify that Karaś's results (summarized in Theorem 1.1) are direct corollaries of our Theorem 3.3. And we also have the following corollaries.

Corollary 3.4. Let $3 \leq p_{1} \leq d_{2} \leq d_{3}$ be integers with $p_{1}$ is a prime number. If $d_{2}$ is odd, then $\left(p_{1}, d_{2}, d_{3}\right) \in \operatorname{mdeg}\left(T\left(k^{3}\right)\right)$ if and only if $p_{1} \mid d_{2}$ or $d_{3} \in p_{1} \mathbb{N}+d_{2} \mathbb{N}$.

Corollary 3.5. Let $5 \leq d_{2} \leq d_{3}$ be integers. Then $\left(5, d_{2}, d_{3}\right) \in \operatorname{mdeg}\left(T\left(k^{3}\right)\right)$ if and only if $5 \mid d_{2}$ or $d_{3} \in 5 \mathbb{N}+d_{2} \mathbb{N}$.

Corollary 3.6. (1) Let $7 \leq d_{2} \leq d_{3}$ be integers with $\left(d_{2}, d_{3}\right) \neq(8,12)$. Then $\left(7, d_{2}, d_{3}\right) \in \operatorname{mdeg}\left(T\left(k^{3}\right)\right)$ if and only if $7 \mid d_{2}$ or $d_{3} \in 7 \mathbb{N}+d_{2} \mathbb{N}$.

(2) Let $11 \leq d_{2} \leq d_{3}$ be integers with $\left(d_{2}, d_{3}\right) \neq(12,18),(14,21),(16,24)$. Then $\left(11, d_{2}, d_{3}\right) \in \operatorname{mdeg}\left(T\left(k^{3}\right)\right)$ if and only if $11 \mid d_{2}$ or $d_{3} \in 11 \mathbb{N}+d_{2} \mathbb{N}$.

Proof. By Theorem 3.3, we may assume that $d_{2}<2 d_{1}-5, d_{2} / \operatorname{gcd}\left(d_{2}, d_{3}\right)=2$ and $d_{3} / \operatorname{gcd}\left(d_{2}, d_{3}\right)=3$. When $d_{1}=7$, this implies that $\left(d_{2}, d_{3}\right)=(8,12)$, and when $d_{1}=11$ this implies that $\left(d_{2}, d_{3}\right)=(12,18),(14,21)$ or $(16,24)$.

In what follows, we relate the research on Conjecture 1.2 to the problem of whether exists a tame automorphism admitting a reduction of type II or III. In fact, we have the following result.

Theorem 3.7. Let $p_{1} \geq 7$ be a prime number and assume that $\left(p_{1}, d_{2}, d_{3}\right)=$ $\left(p_{1}, 2 p_{1}-6,3 p_{1}-9\right) \in \operatorname{mdeg}\left(T\left(k^{3}\right)\right)$. If $p_{1}=7$, i.e. $\left(p_{1}, d_{2}, d_{3}\right)=(7,8,12)$, then there exists a tame automorphism admitting a reduction of type $I I$; if $p_{1}>7$ (in particular if $\left.\left(p_{1}, d_{2}, d_{3}\right)=(11,16,24)\right)$, then there exists a tame automorphism admitting a reduction of type III.

Proof. Notice that $p_{1} \nmid d_{2}$ and $d_{3} \notin p_{1} \mathbb{N}+d_{2} \mathbb{N}$. By Lemma 3.2, there exists a tame automorphism $F$ with mdeg $F=\left(p_{1}, d_{2}, d_{3}\right)$ which admits an elementary reduction. By the proof of Theorem 3.3, there exists some $g(x, y) \in k[x, y]$ such that $\operatorname{deg}\left(F_{1}-g\left(F_{2}, F_{3}\right)\right)<\operatorname{deg} F_{1}$, and thus $\operatorname{deg} g\left(F_{2}, F_{3}\right)=\operatorname{deg} F_{1}=p_{1}$. Let $\operatorname{deg}_{y} g(x, y)=2 q+r$, where $0 \leq r<2$. By Theorem 2.2,

$$
\begin{aligned}
p_{1}=\operatorname{deg} g\left(F_{2}, F_{3}\right) & \geq q\left(2 d_{3}-d_{3}-d_{2}+\operatorname{deg}\left[F_{2}, F_{3}\right]\right)+r d_{3} \\
& =q\left(d_{2} / 2+\operatorname{deg}\left[F_{2}, F_{3}\right]\right)+r d_{3},
\end{aligned}
$$


which implies that $r=0$ and $q \leq 1$. If $q=0$, then $\operatorname{deg}_{y} g(x, y)=0$, and thus $p_{1}=\operatorname{deg} g\left(F_{2}\right) \in d_{2} \mathbb{N}$, a contradiction. Hence $q=1$ and then by (3.4) we obtain $\operatorname{deg}\left[F_{2}, F_{3}\right]<p_{1}<\operatorname{deg} F_{2}+\operatorname{deg} F_{3}$. It follows that $\overline{F_{2}}, \overline{F_{3}}$ are algebraically dependent. In addition, $F_{2}, F_{3}$ are algebraically independent, and the condition $d_{2}=2 p_{1}-6$ and $d_{3}=3 p_{1}-9$ ensures that $\overline{F_{2}} \notin k\left[\overline{F_{3}}\right]$ and $\overline{F_{3}} \notin k\left[\overline{F_{2}}\right]$. Therefore, $F_{2}, F_{3}$ is a 2 -reduced pair.

Now let $\theta=\left(f_{1}, f_{2}, f_{3}\right)=\left(F_{1}, F_{1}+F_{2}, F_{3}\right)$. Then $\operatorname{deg} f_{1}=\operatorname{deg} F_{1}=p_{1}, \operatorname{deg} f_{2}$ $=\operatorname{deg} F_{2}=2 m$ and $\operatorname{deg} f_{3}=\operatorname{deg} F_{3}=3 m$, where $m=p_{1}-3$.

(1) Suppose that $p_{1}=7$. Then $m=4$ and $\left(p_{1}, d_{2}, d_{3}\right)=(7,8,12)$. It follows that $\frac{3}{2} m<\operatorname{deg} f_{1}=p_{1} \leq 2 m$. Notice that $\overline{f_{1}}, \overline{f_{2}}$ are linearly independent. Let $(\alpha, \beta)=(1,0)$ and let $g_{2}:=f_{2}-\alpha f_{1}=F_{2}, g_{3}:=f_{3}-\beta f_{1}=F_{3}$. Then $g_{2}, g_{3}$ is a 2-reduced pair and $\operatorname{deg} g_{2}=2 m, \operatorname{deg} g_{3}=3 m$. Moreover, $\left(f_{1}, g_{2}, g_{3}\right)=\left(F_{1}, F_{2}, F_{3}\right)$ admits an elementary reduction and $\left(g_{1}, g_{2}, g_{3}\right)=\left(f_{1}-g\left(g_{1}, g_{2}\right), g_{2}, g_{3}\right)=\left(F_{1}-\right.$ $\left.g\left(F_{2}, F_{3}\right), F_{2}, F_{3}\right)$ is such a reduction.

Notice that $p_{1}=m+3$. By Lemma 3.1, we have $\operatorname{deg}\left[g_{2}, g_{3}\right] \geq 3$. Then

$$
\begin{aligned}
\operatorname{deg}\left[g_{1}, g_{2}\right] & \leq \operatorname{deg} g_{1}+\operatorname{deg} g_{2} \leq \operatorname{deg} f_{1}-1+\operatorname{deg} g_{2} \\
& =p_{1}-1+2 m=3 m+2<3 m+\operatorname{deg}\left[g_{2}, g_{3}\right] .
\end{aligned}
$$

Therefore, by [SU2, Definition 2], $\theta$ admits a reduction of type II with the active element $f_{1}$.

(2) Suppose that $p_{1}>7$. Since $p$ is a prime number, $p_{1} \geq 11$, and noticing that $p_{1}=m+3$, we have $m<\operatorname{deg} f_{1}=p_{1} \leq \frac{3}{2} m$. Let $(\alpha, \beta, \gamma)=(0,1,0)$ and let $g_{2}:=f_{2}-\beta f_{1}=F_{2}, g_{3}:=f_{3}-\gamma f_{1}-\alpha f_{1}^{2}=F_{3}$. Then $g_{2}, g_{3}$ is a 2-reduced pair and $\operatorname{deg} g_{2}=2 m, \operatorname{deg} g_{3}=3 m$. And $\left(f_{1}, g_{2}, g_{3}\right)=\left(F_{1}, F_{2}, F_{3}\right)$ admits an elementary reduction: $\left(g_{1}, g_{2}, g_{3}\right)=\left(F_{1}-g\left(F_{2}, F_{3}\right), F_{2}, F_{3}\right)$. As proved in $(1), \operatorname{deg}\left[g_{1}, g_{2}\right]<$ $3 m+\operatorname{deg}\left[g_{2}, g_{3}\right]$. Moreover, $\operatorname{deg} g_{1}<\operatorname{deg} f_{1}=p_{1}=m+3 \leq m+\operatorname{deg}\left[g_{2}, g_{3}\right]$. Therefore, by [SU2, Definition 3], $\theta$ admits a reduction of type III with the active element $f_{1}$.

Remark 3.8. If we replace the condition that $p_{1}$ is a prime number by the condition that $p_{1}, d_{2}$ are relatively prime, then Conjecture 1.2 is not valid. In fact, we note that, among other things, Kuroda [Kur1] constructed tame automorphisms with multidegrees $\left(d_{1}, d_{2}, d_{3}\right)=(2 m, 2 p m+p+1,(2 p+1) m)$, where $m=p q+p+q$ and $p, q \in \mathbb{N}-0$. If we take $p=2$, then $\left(d_{1}, d_{2}, d_{3}\right)=(2 m, 4 m+3,5 m)$, where $m=3 q+2$ and $q \in \mathbb{N}-0$. And in this case, $d_{1}, d_{2}$ are relatively prime and $d_{3} \notin d_{1} \mathbb{N}+d_{2} \mathbb{N}$.

Remark 3.9. The editor points out that our Corollaries 3.5 and 3.6(1) were independently obtained by Karaś in [K3, Sections 7.2-7.3], and it was shown 
that the existence of a tame automorphism of $k^{3}$ with multidegree $\left(p_{1}, d_{2}, d_{3}\right):=$ $\left(p_{1}, 2\left(p_{1}-2\right), 3\left(p_{1}-2\right)\right)$ for some prime number $p_{1}>35$ (in particular with multidegree $(37,70,105))$ would imply that the two-dimensional Jacobian conjecture is not true. However, by our Theorem 3.3 , one can see easily that $\left(p_{1}, d_{2}, d_{3}\right)=$ $\left(p_{1}, 2\left(p_{1}-2\right), 3\left(p_{1}-2\right)\right) \notin \operatorname{mdeg}\left(T\left(k^{3}\right)\right)$ for any prime number $p_{1} \geq 3$ because $d_{2}=2\left(p_{1}-2\right) \geq 2 p_{1}-5, p_{1} \nmid d_{2}$ and $d_{3} \notin p_{1} \mathbb{N}+d_{2} \mathbb{N}$.

\section{Acknowledgements}

The first author wishes to thank for the help of Professor Jiping Zhang and for the support of the School of Mathematical Sciences, Peking University.

This work was supported by the Basic Scientific Research Fund of Ministry of Education of China, NSF of China (grant no. 11026039), and the "985 Project" and "211 Project" of Jilin University.

\section{References}

[B] A. Brauer, On a problem on partitions, Amer. J. Math. 64 (1942), 299-312. Zbl 0061.06801 MR 0006196

[E] A. van den Essen, Polynomial automorphisms and the Jacobian Conjecture, Progr. Math. 190, Birkhäuser, Basel, 2000. Zbl 0962.14037 MR 1790619

[EMW] A. van den Essen, L. Makar-Limanov and R. Willems, Remarks on Shestakov-Umirbaev, Report 0414, Radboud Univ. of Nijmegen, 2004.

[J] H. Jung, Über ganze birationale Transformationen der Ebene, J. Reine Angew. Math. 184 (1942), 161-174. Zbl 0027.08503 MR 0008915

[K1] M. Karaś, Tame automorphisms of $\mathbb{C}^{3}$ with multidegree of the form $\left(p_{1}, p_{2}, d_{3}\right)$, Bull. Polish Acad. Sci. Math. 59 (2011), 27-32. Zbl 1215.14059 MR 2810969

[K2] Tame automorphisms of $\mathbb{C}^{3}$ with multidegree of the form $\left(3, d_{2}, d_{3}\right)$, J. Pure Appl. Algebra 214 (2010), 2144-2147. Zbl 1208.14057 MR 2660904

[K3] Multidegrees of tame automorphisms of $\mathbb{C}^{n}$, Dissertationes Math. $477(2011)$, 55 pp.

[Kul] W. van der Kulk, On polynomial rings in two variables, Nieuw Arch. Wisk. 3 (1953), 33-41. Zbl 0050.26002 MR 0054574

[Kur1] S. Kuroda, Automorphisms of a polynomial ring which admit reductions of type I, Publ. RIMS Kyoto Univ. 45 (2009), 907-917. Zbl pre05625042 MR 2569570

[Kur2] Shestakov-Umirbaev reductions and Nagata's conjecture on a polynomial automorphism, Tohoku Math. J. 62 (2010), 75-115. Zbl 1210.14072 MR 2654304

[N] M. Nagata, On the automorphism group of $k[X, Y]$, Lectures in Math 5, Kyoto Univ., Kinokuniya, Tokyo, 1972. Zbl 0306.14001 MR 0337962

[SU1] I. P. Shestakov and U. U. Umirbaev, Poisson brackets and two-generated subalgebras of rings of polynomials, J. Amer. Math. Soc. 17 (2004), 181-196. Zbl 1044.17014 MR 2015333

[SU2] The tame and the wild automorphisms of polynomial rings in three variables, J. Amer. Math. Soc. 17 (2004), 197-227. Zbl 1056.14085 MR 2015334 